\title{
Influenza Virus-Specific Culture
}

National Cancer Institute

\section{Source}

National Cancer Institute. Influenza Virus-Specific Culture. NCI Thesaurus. Code C155815.

Cell culture conditions that are specific for the growth and identification of influenza virus. 